\title{
Existence of nontrivial solutions for $p$-Kirchhoff type equations
}

\section{Chunhan Liu*, Jianguo Wang and Qingling Gao}

*Correspondence: chh-liu1981@163.com Department of Mathematics, Qilu Normal University, Jinan, 250013, P.R. China

\begin{abstract}
In this paper, the linking theorem and the mountain pass theorem are used to show the existence of nontrivial solutions for the $p$-Kirchhoff equations without assuming Ambrosetti-Rabinowitz type growth conditions, nontrivial solutions are obtained.

MSC: 35J60; 35J25
\end{abstract}

Keywords: linking theorem; mountain pass theorem; nontrivial solutions

\section{Introduction}

In this paper, we consider the nonlocal elliptic problem of the $p$-Kirchhoff type given by

$$
\begin{cases}{\left[M\left(\int_{\Omega}|\nabla u|^{p} \mathrm{~d} x\right)\right]^{p-1}\left(-\triangle_{p} u\right)=f(x, u),} & \text { in } \Omega, \\ u=0, & \text { on } \partial \Omega,\end{cases}
$$

where $\Omega \subset R^{N}$ is a bounded domain, and $\triangle_{p} u=\operatorname{div}\left(|\nabla u|^{p-2} \nabla u\right)$ is the $p$-Laplacian with $1<p<N$.

Recently, the equation

$$
\begin{cases}-\left(a+b \int_{\Omega}|\nabla u|^{2} \mathrm{~d} x\right) \Delta u=f(x, u), & \text { in } \Omega, \\ u=0, & \text { on } \partial \Omega,\end{cases}
$$

began to attract the attention of several researchers only after Lion [1] had proposed an abstract framework for this problem. Perera and Zhang [2] obtained a nontrivial solution of (2) by using the Yang index and critical group. They revisited (2) via invariant sets of decent flow and obtained the existence of a positive solution, a negative, and a sign-changing solutions in [3].

The study of Kirchhoff-type equations has been extended to the following case involving the $p$-Laplacian:

$$
\begin{cases}-M\left(\frac{1}{p} \int_{\Omega}|\nabla u|^{p} \mathrm{~d} x\right) \operatorname{div}\left(|\nabla u|^{p-2} \nabla u\right)=f(x, u), & \text { in } \Omega, \\ u=0, & \text { on } \partial \Omega,\end{cases}
$$

for details see [4-6]. One of the authors has done some related work on this field. Liu [7] gave infinite solutions to the following equation via the fountain theorem and the dual fountain theorem:

$$
\begin{cases}{\left[M\left(\int_{\Omega}\left(|\nabla u|^{p}+\lambda(x)|u|^{p}\right) \mathrm{d} x\right)\right]^{p-1}\left(-\triangle_{p} u+\lambda(x)|u|^{p-2} u\right)=f(x, u),} & \text { in } \Omega, \\ |\nabla u|^{p-2} \frac{\partial u}{\partial \nu}=\eta|u|^{p-2}, & \text { on } \partial \Omega .\end{cases}
$$

@2013 Liu et al.; licensee Springer. This is an Open Access article distributed under the terms of the Creative Commons Attribution License (http://creativecommons.org/licenses/by/2.0), which permits unrestricted use, distribution, and reproduction in any medium, provided the original work is properly cited. 
However, to the best of our knowledge, there have been few papers dealing with equation (1) using the linking theorem and the mountain pass theorem. This paper will make some contribution to this research field.

It is well known (see [8]) that the eigenvalue problem

$$
\begin{cases}-\triangle_{p} u=\lambda|u|^{p-2} u & \text { in } \Omega \\ u=0 & \text { on } \partial \Omega\end{cases}
$$

has the first eigenvalue $\lambda_{1}>0$, which is simple, and has an associated eigenfunction $\phi_{1}>0$. It is also known that $\lambda_{1}$ is an isolated point of $\sigma\left(-\triangle_{p}\right)$, the spectrum of $-\triangle_{p}$, which contains at least an eigenvalue sequence $\left\{\lambda_{n}\right\}$ and $0<\lambda_{1}<\lambda_{2} \leq \lambda_{3} \leq \cdots \leq \lambda_{n} \rightarrow \infty$.

Let

$$
W_{0}^{1, p}(\Omega)=\left\{u \in L^{p}(\Omega): \int_{\Omega}|\nabla u|^{p} \mathrm{~d} x<\infty \text { and }\left.u\right|_{\partial \Omega}=0\right\}
$$

be a Banach space with the norm $\|u\|=\|u\|_{p}=\left(\int_{\Omega}|\nabla u|^{p} \mathrm{~d} x\right)^{\frac{1}{p}}$ for $u \in W_{0}^{1, p}(\Omega) . W=$ $\operatorname{span}\left\{\phi_{1}\right\}$ be the one-dimensional eigenspace associated with $\lambda_{1}$, where $\left\|\phi_{1}\right\|=1$. Let $W_{0}^{1, p}(\Omega)=W \oplus V$, where $V=\left\{u \in W_{0}^{1, p}(\Omega): \int_{\Omega} u \phi_{1}^{p-1} \mathrm{~d} x=0\right\}$, there exists $\bar{\lambda}>\lambda_{1}$ such that

$$
\int_{\Omega}|\nabla u|^{p} \mathrm{~d} x \geq \bar{\lambda} \int_{\Omega}|u|^{p} \mathrm{~d} x \quad \text { for } u \in V
$$

When $p=2$, we can take $\bar{\lambda}=\lambda_{2}$, the second eigenvalue of $-\triangle$ in $H_{0}^{1}(\Omega)$.

In this paper, the weak solutions of (1) are the critical points of the energy functional

$$
\Phi(u)=\frac{1}{p} \hat{M}\left(\int_{\Omega}|\nabla u|^{p} \mathrm{~d} x\right)-\int_{\Omega} F(x, u) \mathrm{d} x,
$$

where $\hat{M}(u)=\int_{0}^{u}[M(s)]^{p-1} \mathrm{~d} s, F(x, u)=\int_{0}^{u} f(x, s) \mathrm{d} s$. Obviously, $\Phi(u) \in C^{1}\left(W_{0}^{1, p}(\Omega), R\right)$ and for all $u, v \in W_{0}^{1, p}(\Omega)$,

$$
\left\langle\Phi^{\prime}(u), v\right\rangle=\left[M\left(\int_{\Omega}|\nabla u|^{p} \mathrm{~d} x\right)\right]^{p-1} \int_{\Omega}\left(|\nabla u|^{p-2} \nabla u \nabla v\right) \mathrm{d} x-\int_{\Omega} f(x, u) v \mathrm{~d} x .
$$

In this paper we use the following notation: $L^{p}(\Omega)$ denotes the Lebesgue space with the norm $|\cdot|_{p} ;|\Omega|$ denotes the Lebesgue measure of the set $\Omega \subset R^{N} ;\langle\cdot, \cdot\rangle$ is the dual pairing of the space $\left(W_{0}^{1, p}(\Omega)\right)^{*}$ and $W_{0}^{1, p}(\Omega) ; \rightarrow$ (resp. $\rightarrow$ ) denotes strong (resp. weak) convergence. $C_{0}, C_{1}, C_{2}, \ldots$ denote positive constants (possibly different).

Definition 1 [9] Let $\Phi \in C^{1}(X, R)$, we say that $\Phi$ satisfies the Cerami condition at the level $c \in R$ if any sequence $\left\{u_{n}\right\} \subset X$, along with

$$
\Phi\left(u_{n}\right) \rightarrow c \text { and }\left(1+\left\|u_{n}\right\|\right) \Phi^{\prime}\left(u_{n}\right) \rightarrow 0 \quad \text { as } n \rightarrow \infty,
$$

possesses a convergent subsequence; $\Phi$ satisfies the $(C)$ condition if $\Phi$ satisfies $(C)_{c}$ for all $c \in R$. 
Definition 2 [9] A subset $A$ of $E$ is link (with respect to $\Phi$ ) to $B$ of $E$ if $A \cap B=\emptyset$, for every $\Gamma \in \Phi$, there is $t \in[0,1]$ such that $\Gamma(t, A) \cap B \neq \emptyset$.

Theorem 1 [9] (Linking theorem) Let $X=Y \oplus Z$ be a Banach space with $\operatorname{dim} Y<\infty$. Let $\rho>r>0$, and let $z \in Z$ be such that $\|u\|=r$. Define

$$
\begin{aligned}
& M=\{u=y+\lambda z:\|u\| \leq \rho, \lambda \geq 0, y \in Y\}, \\
& M_{0}=\{u=y+\lambda z: y \in Y,\|u\|=\rho \text { and } \lambda \geq 0 \text { or }\|u\| \leq \rho \text { and } \lambda=0\}, \\
& N=\{u \in Z:\|u\|=r\} .
\end{aligned}
$$

Let $\Phi \in C^{1}(X, R)$ be such that

$$
b=\inf _{N} \Phi>a=\max _{M_{0}} \Phi .
$$

If $\Phi$ satisfies the (PS) condition with

$$
\begin{aligned}
& c=\inf _{\gamma \in \Gamma} \max _{u \in M} \Phi(\gamma(u)), \\
& \Gamma=\left\{\gamma \in C(M, X):\left.\gamma\right|_{M_{0}}=i d\right\},
\end{aligned}
$$

then $c$ is a critical value of $\Phi$.

Remark 1 If $\Phi$ satisfies the $(C)$ condition, then Theorem 1 still holds.

Theorem 2 [10] (Mountain pass theorem) Let $X$ be a real Banach space, and let $\Phi \in$ $C^{1}(X, R)$ satisfy the $(C)$ condition. Suppose, for some $\alpha<\beta, \rho>0$ and $u \in X,\|u\|>\rho$,

$$
\max \{\Phi(0), \Phi(u)\} \leq \alpha<\beta \leq \inf _{\|u\|=\rho} \Phi(u) .
$$

Then $\Phi$ has a critical value $c \geq \beta>0$ characterized by

$$
c=\inf _{\gamma \in \Gamma} \max _{\tau \in[0,1]} \Phi(\gamma(\tau)),
$$

where

$$
\Gamma=\{\gamma \in C([0,1], X): \gamma(0)=0, \gamma(1)=u\} .
$$

\section{Main results}

In this section, we give our main theorem. Near the origin, we make the following assumptions.

Suppose that $M: R^{+} \rightarrow R^{+}$is a continuous function satisfying the following conditions:

$\left(m_{0}\right)$ there exists a constant $m_{0}>0$ such that $M(t) \geq m_{0}$ for all $t \geq 0$;

$\left(m_{1}\right)$ there exists a constant $m_{1}>0$ such that $M(t) \leq m_{1}$ for all $t \geq 0$ and $\hat{M}(t) \geq$ $a[M(t)]^{p-1} t, a>1$.

Caratheodory function $f$ satisfies: 
$\left(F_{0}\right)$ For some $p<q<p^{*}=\frac{N p}{N-p}$, there exists a constant $C>0$ such that

$$
|f(x, t)| \leq C\left(1+|t|^{q-1}\right) \quad \text { for all } x \in \Omega, t \in R .
$$

$\left(F_{1}\right)$ There exist $0<\alpha<m_{0}^{p-1} \lambda_{1}(a-1)$ and $\sigma \in[0, p]$ such that

$$
\liminf _{|t| \rightarrow \infty} \frac{f(x, t) t-p F(x, t)}{|t|^{\sigma}}>-\alpha \quad \text { uniformly in } x \in \Omega \text {. }
$$

$\left(F_{2}\right) \quad \lim _{|t| \rightarrow \infty} \frac{p F(x, t)}{|t|^{p}}=+\infty$ uniformly in $x \in \Omega$.

$\left(F_{2}\right)^{\prime} \liminf _{|t| \rightarrow \infty} \frac{p F(x, t)}{|t|^{p}}>m_{1}^{p-1} \lambda_{1}$ uniformly in $x \in \Omega$.

$\left(F_{3}\right) \quad \lim \sup _{|t| \rightarrow 0} \frac{p F(x, t)}{|t|^{p}}<m_{0}^{p-1} \bar{\lambda}$ uniformly in $x \in \Omega$.

$\left(F_{3}\right)^{\prime} \lim \sup _{|t| \rightarrow 0} \frac{p F(x, t)}{|t|^{p}}<m_{0}^{p-1} \lambda_{1}$ uniformly in $x \in \Omega$.

$\left(F_{4}\right) \quad F(x, t) \geq \frac{m_{1}^{p-1} \lambda_{1}}{p}|t|^{p}, \forall(x, t) \in \Omega \times R^{1}$.

The main results of this paper are the following.

Theorem 3 Assume that $\left(m_{0}\right),\left(m_{1}\right)$ and $\left(F_{0}\right)-\left(F_{4}\right)$ hold, then problem (1) has at least one nontrivial weak solution in $W_{0}^{1, p}(\Omega)$.

Theorem 4 Assume that $\left(m_{0}\right),\left(m_{1}\right)$ and $\left(F_{0}\right),\left(F_{1}\right),\left(F_{2}\right)^{\prime},\left(F_{3}\right)^{\prime}$ hold, then problem $(1)$ has at least one nontrivial weak solution in $W_{0}^{1, p}(\Omega)$.

\section{Proofs of theorems}

First, we give several lemmas.

Lemma 1 [7] Under assumptions $\left(m_{0}\right)$ and $\left(F_{0}\right)$, any bounded sequence $\left\{u_{n}\right\} \subset W_{0}^{1, p}(\Omega)$ such that $\Phi^{\prime}(u) \rightarrow 0$ in $\left(W_{0}^{1, p}(\Omega)\right)^{*}$ as $n \rightarrow \infty$ has a convergent subsequence.

Lemma 2 Under assumptions $\left(m_{0}\right)$ and $\left(F_{1}\right)$, the functional $\Phi(u)$ satisfies the $(C)$ condition.

Proof Let $\left\{u_{n}\right\} \subset W_{0}^{1, p}(\Omega)$, for every $c>0$,

$$
\Phi\left(u_{n}\right) \rightarrow c \text { and }\left(1+\left\|u_{n}\right\|\right) \Phi^{\prime}\left(u_{n}\right) \rightarrow 0 \quad \text { as } n \rightarrow \infty
$$

We claim that $\left\{u_{n}\right\}$ is bounded in $W_{0}^{1, p}(\Omega)$. For this purpose, we can suppose that $\left\|u_{n}\right\| \rightarrow$ $\infty$. By $\left(F_{1}\right)$, there exists $r>1$ such that

$$
f(x, t) t-p F(x, t) \geq-\alpha|t|^{\sigma}, \quad \forall|t|>r .
$$

For large $n$, set $\Omega_{n}=\{x \in \Omega:|u|>r\}$, (3) and (4) imply that there exists $M_{1}>0$ such that

$$
\begin{aligned}
p(1+c) \geq & p \Phi\left(u_{n}\right)-\left\langle\Phi^{\prime}\left(u_{n}\right), u_{n}\right\rangle \\
= & \hat{M}\left(\int_{\Omega}\left|\nabla u_{n}\right|^{p} \mathrm{~d} x\right)-\int_{\Omega} p F\left(x, u_{n}\right) \mathrm{d} x \\
& -\left[\left[M\left(\int_{\Omega}\left|\nabla u_{n}\right|^{p} \mathrm{~d} x\right)\right]^{p-1} \int_{\Omega}\left(\left|\nabla u_{n}\right|^{p}\right) \mathrm{d} x-\int_{\Omega} f\left(x, u_{n}\right) u_{n} \mathrm{~d} x\right]
\end{aligned}
$$




$$
\begin{aligned}
\geq & (a-1) m_{0}^{p-1}\left\|u_{n}\right\|^{p}+\int_{\Omega}\left[f\left(x, u_{n}\right) u_{n}-p F\left(x, u_{n}\right)\right] \mathrm{d} x \\
= & (a-1) m_{0}^{p-1}\left\|u_{n}\right\|^{p}+\int_{\Omega_{n}}\left[f\left(x, u_{n}\right) u_{n}-p F\left(x, u_{n}\right)\right] \mathrm{d} x \\
& +\int_{\Omega \backslash \Omega_{n}}\left[f\left(x, u_{n}\right) u_{n}-p F\left(x, u_{n}\right)\right] \mathrm{d} x \\
\geq & (a-1) m_{0}^{p-1}\left\|u_{n}\right\|^{p}-\int_{\Omega_{n}} \alpha\left|u_{n}\right|^{\sigma} \mathrm{d} x-M_{1} \\
\geq & (a-1) m_{0}^{p-1}\left\|u_{n}\right\|^{p}-\int_{\Omega_{n}} \alpha\left|u_{n}\right|^{p} \mathrm{~d} x-M_{1} \\
\geq & {\left[(a-1) m_{0}^{p-1}-\frac{\alpha}{\lambda_{1}}\right]\left\|u_{n}\right\|^{p}-M_{1} . }
\end{aligned}
$$

This is a contradiction. Then $\left\{u_{n}\right\}$ is bounded in $W_{0}^{1, p}(\Omega)$. By Lemma 1 , we see that $\left\{u_{n}\right\}$ has a convergent subsequence in $W_{0}^{1, p}(\Omega)$.

Proof of Theorem 3 We obtain from assumptions $\left(m_{0}\right),\left(F_{0}\right)$ and $\left(F_{3}\right)$ that for some $\varepsilon>0$ small, there exists $C_{1}>0$ such that

$$
F(x, u) \leq \frac{m_{0}^{p-1}(\bar{\lambda}-\varepsilon)}{p}|u|^{p}+C_{1}|u|^{q}, \quad \forall x \in \Omega .
$$

Taking $u \in V$, using the inequality $\int_{\Omega}|\nabla u|^{p} \mathrm{~d} x \geq \bar{\lambda} \int_{\Omega}|u|^{p} \mathrm{~d} x$ and the Sobolev inequality $|u|_{q}^{q} \leq K\|u\|^{q}$, we have

$$
\begin{aligned}
\Phi(u) & =\frac{1}{p} \hat{M}\left(\int_{\Omega}|\nabla u|^{p} \mathrm{~d} x\right)-\int_{\Omega} F(x, u) \mathrm{d} x \\
& \geq \frac{m_{0}^{p-1}}{p}\|u\|^{p}-\frac{m_{0}^{p-1}(\bar{\lambda}-\varepsilon)}{p} \int_{\Omega}|u|^{p} \mathrm{~d} x-C_{1} \int_{\Omega}|u|^{q} \mathrm{~d} x \\
& \geq \frac{m_{0}^{p-1}}{p}\|u\|^{p}-\frac{m_{0}^{p-1}(\bar{\lambda}-\varepsilon)}{p \bar{\lambda}}\|u\|^{p}-C_{1} K\|u\|^{q} \\
& =\frac{m_{0}^{p-1}}{p} \frac{\varepsilon}{\bar{\lambda}}\|u\|^{p}-C_{1} K\|u\|^{q} .
\end{aligned}
$$

Then there exists $r>0$ such that $b=\inf _{u \in V,\|u\|=r} \Phi(u)>0$.

Let $z \in V,\|z\|=r$ and $M_{0}=\{u=v+\lambda z: v \in W:\|u\|=\rho$ and $\lambda \geq 0$, or $\|u\| \leq \rho$ and $\lambda=$ $0\}, N=\{u \in V:\|u\|=r\}$.

For every $u \in M_{0}$, if $u=v+\lambda z,\|u\| \leq \rho$ and $\lambda=0$, then $u=v \in W$. By $\left(F_{4}\right)$, we know that

$$
\begin{aligned}
\Phi(u) & =\frac{1}{p} \hat{M}\left(\int_{\Omega}|\nabla u|^{p} \mathrm{~d} x\right)-\int_{\Omega} F(x, u) \mathrm{d} x \\
& \leq \frac{m_{1}^{p-1}}{p}\|u\|^{p}-\int_{\Omega} F(x, u) \mathrm{d} x \\
& \leq \frac{m_{1}^{p-1}}{p} \lambda_{1} \int_{\Omega}|u|^{p} \mathrm{~d} x-\int_{\Omega} F(x, u) \mathrm{d} x \\
& \leq \int_{\Omega}\left[\frac{m_{1}^{p-1}}{p} \lambda_{1}|u|^{p} \mathrm{~d} x-F(x, u)\right] \mathrm{d} x \leq 0 .
\end{aligned}
$$


Since $\operatorname{dim} W \oplus R z<\infty$, there exists $C_{0}>1$ such that

$$
\|u\|^{p} \leq C_{0}|u|_{p}^{p}, \quad \forall u \in W \oplus R z
$$

By $\left(F_{0}\right)$ and $\left(F_{2}\right)$, there exists $\rho>0$ such that $F(x, u) \geq \frac{m_{1}^{p-1}}{p} C_{0}^{2}|u|^{p}, \forall|u|>\rho$. Let $M=$ $\max \left\{0, \inf _{|u| \leq \rho} F(x, u)\right\}$, then we have

$$
F(x, u) \geq \frac{m_{1}^{p-1}}{p} C_{0}^{2}|u|^{p}-M
$$

Therefore, $\forall u \in W \oplus R z$, we have

$$
\begin{aligned}
\Phi(u) & =\frac{1}{p} \hat{M}\left(\int_{\Omega}|\nabla u|^{p} \mathrm{~d} x\right)-\int_{\Omega} F(x, u) \mathrm{d} x \\
& \leq \frac{m_{1}^{p-1}}{p}\|u\|^{p}-\frac{m_{1}^{p-1} C_{0}^{2}}{p} \int_{\Omega}|u|^{p} \mathrm{~d} x+M|\Omega| \\
& \leq \frac{m_{1}^{p-1}}{p}\|u\|^{p}-\frac{m_{1}^{p-1} C_{0}}{p}\|u\|^{p}+M|\Omega| \\
& \leq-\frac{\left(C_{0}-1\right) m_{1}^{p-1}}{p}\|u\|^{p}+M|\Omega| .
\end{aligned}
$$

Hence, for $\rho=\|u\|$ large enough, we have $\Phi(u) \rightarrow-\infty$.

Then there exists $\rho>r>0$ such that

$$
\inf _{N} \Phi(u)>0=\max _{M_{0}} \Phi(u)
$$

By Lemmas 1 and 2, $\Phi$ satisfies the $(C)$ condition. Then the conclusion follows from Theorem 1 and Remark 1.

Remark 2 (i) There exists $R>0$ such that $p F(x, u) \leq u f(x, u), \forall|u| \geq R, x \in \Omega$, which implies $\forall|u| \geq R, x \in \Omega$,

$$
f(x, u) u-p F(x, u) \geq 0>-\alpha|u|^{\sigma} .
$$

Then

$$
\liminf _{|t| \rightarrow \infty} \frac{f(x, t) t-p F(x, t)}{|t|^{\sigma}}>-\alpha \quad \text { uniformly in } x \in \Omega .
$$

Hence $\left(F_{1}\right)$ is much weaker than Ambrosetti-Rabinowitz type growth conditions.

(ii) If $f(x, u) u-p F(x, u) \rightarrow+\infty$, as $|u| \rightarrow \infty$ uniformly in $x \in \Omega$, then

$$
\liminf _{|t| \rightarrow \infty} \frac{f(x, t) t-p F(x, t)}{|t|^{\sigma}} \geq 0>-\alpha \quad \text { uniformly in } x \in \Omega \text {. }
$$

\section{Example 1 Set}

$$
f(x, t)= \begin{cases}0, & t<0 \\ m_{0}^{p-1} \tilde{\lambda} t^{p-1}, & 0 \leq t \leq 1 \\ t^{p-1} \ln t+m_{0}^{p-1} \tilde{\lambda} t^{p-1}, & t>1\end{cases}
$$


where $\lambda_{1}<\tilde{\lambda}<\bar{\lambda}, m_{1}^{p-1} \lambda_{1}+1<m_{0}^{p-1} \tilde{\lambda}$. Then it is easy to verify that $f(x, t)$ satisfies $\left(F_{0}\right)-\left(F_{4}\right)$ with $\sigma=p$. When $t<0$, we can use odd expansion to $f(x, t)$.

\section{Example 2 Set}

$$
f(x, t)= \begin{cases}0, & t<0 \\ m_{0}^{p-1} \tilde{\lambda} t^{p-1}, & 0 \leq t<2 \pi \\ \frac{m_{1}^{p-1} \lambda_{1}}{p}\left[q^{\prime}(x, t) t^{p}+p q(x, t) t^{p-1}\right]+m_{0}^{p-1} \tilde{\lambda} t^{p-1}, & t \geq 2 \pi\end{cases}
$$

where

$$
q^{\prime}(x, t)=\left\{\begin{array}{ll}
t \sin t, & t \in[2 k \pi,(2 k+1) \pi) \\
\frac{\sin t}{t}, & t \in[(2 k+1) \pi,(2 k+2) \pi)
\end{array} \quad(k=1,2, \ldots)\right.
$$

$q(x, t)=\int_{2 \pi}^{t} q^{\prime}(x, s) \mathrm{d} s, \lambda_{1}<\tilde{\lambda}<\bar{\lambda}, m_{1}^{p-1} \lambda_{1}<m_{0}^{p-1} \tilde{\lambda}$. Then it is easy to verify that $f(x, t)$ satisfies $\left(F_{0}\right)-\left(F_{4}\right)$ with $\sigma=p$. When $t<0$, we can use odd expansion to $f(x, t)$.

Proof of Theorem 4 We obtain from assumptions $\left(m_{0}\right),\left(F_{0}\right),\left(F_{3}\right)^{\prime}$ that for some $\varepsilon>0$ small, there exists $C_{2}>0$ such that

$$
F(x, u) \leq \frac{m_{0}^{p-1}}{p}\left(\lambda_{1}-\varepsilon\right)|u|^{p}+C_{2}|u|^{q} \quad \text { for all } x \in \Omega
$$

Taking $u \in W_{0}^{1, p}(\Omega)$, using the inequality $\int_{\Omega}|\nabla u|^{p} \mathrm{~d} x \geq \lambda_{1} \int_{\Omega}|u|^{p} \mathrm{~d} x$ and the Sobolev inequality $|u|_{q}^{q} \leq K\|u\|^{q}$, we have

$$
\begin{aligned}
\Phi(u) & =\frac{1}{p} \hat{M}\left(\int_{\Omega}|\nabla u|^{p} \mathrm{~d} x\right)-\int_{\Omega} F(x, u) \mathrm{d} x \\
& \geq \frac{m_{0}^{p-1}}{p}\|u\|^{p}-\frac{m_{0}^{p-1}\left(\lambda_{1}-\varepsilon\right)}{p} \int_{\Omega}|u|^{p} \mathrm{~d} x-C_{2} \int_{\Omega}|u|^{q} \mathrm{~d} x \\
& \geq \frac{m_{0}^{p-1}}{p}\|u\|^{p}-\frac{m_{0}^{p-1}\left(\lambda_{1}-\varepsilon\right)}{p \lambda_{1}}\|u\|^{p}-C_{2} K\|u\|^{q} \\
& =\frac{m_{0}^{p-1}}{p} \frac{\varepsilon}{\lambda_{1}}\|u\|^{p}-C_{2} K\|u\|^{q} .
\end{aligned}
$$

Then there exists $\rho>0$ such that $\inf _{\|u\|=\rho} \Phi(u)>0$.

By $\left(F_{0}\right)$ and $\left(F_{2}\right)^{\prime}$, there exist $\beta>\lambda_{1}, \varepsilon>0, \rho>0$, and $\beta-\varepsilon>\lambda_{1}$ such that $|u|>\rho$,

$$
F(x, u) \geq \frac{m_{1}^{p-1}(\beta-\varepsilon)}{p}|u|^{p} .
$$

Let $M_{1}=\max \left\{0, \inf _{\|u\| \leq \rho} F(x, u)\right\}$, then we have

$$
F(x, u) \geq \frac{m_{1}^{p-1}(\beta-\varepsilon)}{p}|u|^{p}-M_{1}
$$


For $\forall u \in W$, we have

$$
\begin{aligned}
\Phi(u) & =\frac{1}{p} \hat{M}\left(\int_{\Omega}|\nabla u|^{p} \mathrm{~d} x\right)-\int_{\Omega} F(x, u) \mathrm{d} x \\
& \leq \frac{m_{1}^{p-1}}{p}\|u\|^{p}-\frac{m_{1}^{p-1}(\beta-\varepsilon)}{p} \int_{\Omega}|u|^{p} \mathrm{~d} x+M_{1}|\Omega| .
\end{aligned}
$$

Therefore, for $\beta-\varepsilon>\lambda_{1}$, let $u=t \phi_{1}, t \in R$, we have

$$
\begin{aligned}
\Phi\left(t \phi_{1}\right) & \leq \frac{m_{1}^{p-1}}{p}|t|^{p}-\frac{m_{1}^{p-1}(\beta-\varepsilon)}{p} \int_{\Omega}\left|t \phi_{1}\right|^{p} \mathrm{~d} x+M_{1}|\Omega| \\
& \leq \frac{m_{1}^{p-1}}{p}|t|^{p}-\frac{m_{1}^{p-1}(\beta-\varepsilon)}{p \lambda_{1}}|t|^{p}+M_{1}|\Omega| \\
& =\frac{m_{1}^{p-1}}{p}\left(1-\frac{\beta-\varepsilon}{\lambda_{1}}\right)|t|^{p}+M_{1}|\Omega| \rightarrow-\infty \text { as } t \rightarrow \infty .
\end{aligned}
$$

Hence there exists $u_{1}=t_{1} \phi_{1} \in W_{0}^{1, p}(\Omega),\left\|u_{1}\right\|>\rho$ such that $\Phi\left(u_{1}\right) \leq 0$.

Then

$$
\inf _{\|u\|=\rho} \Phi(u)>0 \geq \max \left\{\Phi(0), \Phi\left(u_{1}\right)\right\}
$$

Summing up Lemma 1 and Lemma 2, $\Phi(u)$ satisfies all the conditions of Theorem 2, then the conclusion follows from Theorem 2.

Remark 3 The result of Theorem 1.1 in [11] corresponds to our results for the case $m_{0}=$ $m_{1}=1$ and $\left(F_{2}^{+}\right)_{\mu}$ replaces $\left(F_{1}\right)$. It is easy to see that $\left(F_{1}\right)$ is much weaker than $\left(F_{2}^{+}\right)_{\mu}$, hence the results of Theorems 3 and 4 extend the results of [11].

Example 3 Set

$$
f(x, t)= \begin{cases}0, & t<0 \\ m_{0}^{p-1} \mu_{2} t^{p-1}, & 0 \leq t \leq 1 \\ m_{1}^{p-1} \mu_{1} t^{p-1}-m_{1}^{p-1} \mu_{1}+m_{0}^{p-1} \mu_{2} t, & t>1\end{cases}
$$

where $p$ is odd, $\mu_{1}>\lambda_{1}>\mu_{2}$,

$$
f(x, t)= \begin{cases}m_{1}^{p-1} \mu_{1} t^{p-1}+m_{1}^{p-1} \mu_{1}+m_{0}^{p-1} \mu_{2} t, & t<-1 \\ m_{0}^{p-1} \mu_{2} t^{p-1}, & |t| \leq 1 \\ m_{1}^{p-1} \mu_{1} t^{p-1}-m_{1}^{p-1} \mu_{1}+m_{0}^{p-1} \mu_{2} t, & t>1\end{cases}
$$

where $p$ is even, $\mu_{1}>\lambda_{1}>\mu_{2}$.

Then similar to [12], it is easy to verify that $f(x, t)$ satisfies $\left(F_{0}\right),\left(F_{1}\right),\left(F_{2}\right)^{\prime},\left(F_{3}\right)^{\prime}$ with $\sigma=p$. 
Authors' contributions

$\mathrm{CL}$ and JW obtained the results in a joint research. All the authors read and approved the final manuscript.

\section{Acknowledgements}

This work was supported by the National Nature Science Foundation of China (10971179) and the Project of Shandong Province Higher Educational Science and Technology Program (J09LA55, J12LI53).

Received: 13 July 2013 Accepted: 29 November 2013 Published: 30 Dec 2013

\section{References}

1. Lions, JL: On some equations in boundary value problems of mathematical physics. In: Contemporary Developments in Continuum Mechanics and Partial Differential Equations. Proc. Internat. Sympos., Inst. Mat. Univ. Fed., Rio de Janeiro, 1977. North-Holland Math. Stud., vol. 30, pp. 284-346. North-Holland, Amsterdam (1978)

2. Perera, K, Zhang, Z: Nontrivial solutions of Kirchhoff-type problems via the Yang index. J. Differ. Equ. 221(1), 246-255 (2006)

3. Zhang, Z, Perera, K: Sign changing solutions of Kirchhoff type problems via invariant sets of descent flow. J. Math. Anal. Appl. 317(2), 456-463 (2006)

4. Corrêa, FJSA, Figueiredo, GM: On a elliptic equation of $p$-Kirchhoff type via variational methods. Bull. Aust. Math. Soc. 74, 263-277 (2006)

5. Dreher, M: The Kirchhoff equation for the $p$-Laplacian. Rend. Semin. Mat. (Torino) 64, 217-238 (2006)

6. Dreher, M: The ware equation for the $p$-Laplacian. Hokkaido Math. J. 36, 21-52 (2007)

7. Liu, D: On a $p$-Kirchhoff equation via Fountain theorem and dual Fountain theorem. Nonlinear Anal. 72, $302-308$ (2010)

8. Lindqvist, P. On the equation $\operatorname{div}\left(|\nabla u|^{p-2} \nabla u\right)+\lambda|u|^{p-1} u=0$. Proc. Am. Math Soc 109, 609-623 (1990)

9. Willem, M: Minimax Theorems. Birkhäuser, Boston (1996)

10. Schechter, M: A variation of the mountain pass lemma and applications. J. Lond. Math. Soc. 44(3), 491-502 (1991)

11. Costa, DG, Magalhaes, CA: Existence results for perturbations of the $p$-Laplacian. Nonlinear Anal. TMA 24, 409-418 (1995)

12. Cheng, B: New existence and multiplicity of nontrivial solutions for nonlocal elliptic Kirchhoff type problems. J. Math. Anal. Appl. 394, 488-495 (2012)

10.1186/1687-2770-2013-279

Cite this article as: Liu et al.: Existence of nontrivial solutions for $p$-Kirchhoff type equations. Boundary Value Problems 2013, 2013:279

\section{Submit your manuscript to a SpringerOpen ${ }^{\circ}$ journal and benefit from:}

- Convenient online submission

- Rigorous peer review

Immediate publication on acceptance

- Open access: articles freely available online

- High visibility within the field

- Retaining the copyright to your article 